\title{
Chronic Olanzapine or Sertindole Treatment Results in Reduced Oral Chewing Movements in Rats Compared to Haloperidol
}

Xue-Min Gao, M.D., Kazuo Sakai, M.D., and Carol A. Tamminga, M.D.

Chronic haloperidol treatment typically produces late-onset, purposeless oral chewing movements in laboratory rats with a prevalence of 40 to $60 \%$. Chronic clozapine does not produce these movements. Based on the phenomenologic and pharmacologic similarities between these rat chewing movements and human tardive dyskinesia (TD), the animal movements are often used as a model of tardive dyskinesia (TD). Here we report results of the association of oral chewing movements in rats with chronic administration of two new antipsychotic drugs, olanzapine and sertindole. Because each of these antipsychotic drugs has a very low incidence of acute Parkinsonism in human studies, they are candidates for showing a low tardive dyskinesia risk.

Neither new drug produced a significant incidence of haloperidol-like chewing in rats, nor did movement ratings after their chronic administration differ from placebo; whereas, haloperidol produced a $60 \%$ prevalence of purposeless chewing and a prevalence significantly increased from placebo. This low rate of oral dyskinesias in rats is consistent with several of the preclinical characteristics of the drugs and correlates with their low acute motor side effects in clinical trials. We propose, although have not yet tested in humans, that these animal results will predict low TD liability of these drugs.

[Neuropsychopharmacology 19:428-433, 1998]

(C) 1998 American College of Neuropsychopharmacology.

Published by Elsevier Science Inc.
KEY WORDS: Dyskinesia; Olanzapine; Sertindole;

Haloperidol; Tardive dyskinesia; Chronic neuroleptic treatment

Traditional antipsychotics, such as haloperidol or fluphenazine, induce purposeless oral chewing movements in rats when they are administered chronically (Clow et al. 1980; Ellison et al. 1987; Waddington 1990; Glenthoj and Hemmingsen 1989). These dyskinesias appear gradually, and they occur in only some of the animals. Because characteristics of these neuroleptic-induced rat

From the Maryland Psychiatric Research Center (X-MG, KS, CAT), University of Maryland School of Medicine, UMAB, Baltimore, Maryland, USA.

Address correspondence to: Dr. C.A. Tamminga, University of Maryland School of Medicine, Maryland Psychiatric Research Center, Baltimore, MD 21228, USA.

Received October 6, 1997; accepted April 20, 1998. movements parallel certain features (although not all) of the human neuroleptic-induced dyskinetic syndrome tardive dyskinesia (TD), they are often used in its study.

General criteria for using an animal preparation as a model for a human condition have previously been proposed (McKinney and Bunney 1969) and refined (Weiss and Kilts 1995). Scientists have stressed that the most critical elements for comparison are similarities in etiology, phenomenology, biochemistry, and pharmacology between the animal and the human condition. The etiology of these rat oral dyskinesias (i.e., the chronic antipsychotic treatment) matches the etiology of TD, as do some of the demographic aspects of the rat movements, such as slow symptom onset, delayed offset, and jerky quality (Tamminga et al. 1990; Waddington 1990). Moreover, whereas traditional neuroleptics cause these purposeless movements in rats and tardive dyskinesia in humans, clozapine fails to produce dyskinesias in ei- 
ther rats or humans (Gunne et al. 1982; Kakigi et al. 1995).

The difficulty of distinguishing types of antipsychotic-induced movements in rats, along with the limited oral distribution of the movements in rats, and disagreements about the anticholinergic suppressability of the rat movements, have maintained a proper skepticism about using this preparation without reservation as an animal model of human TD. In this report, we have attempted to expand the pharmacology of these rat dyskinesias by testing whether two of the new antipsychotic drugs, olanzapine and sertindole, which have known low acute motor side effect profiles in humans, would induce purposeless oral chewing in rats.

Olanzapine is a new antipsychotic drug developed by Lilly Laboratories, Inc., a thio[1,5]benzo-diazepine (Bymaster et al. 1996). It has potent antipsychotic actions in schizophrenic psychosis, comparable to traditional neuroleptics, and is active against both positive and negative symptoms (Beasley et al. 1996). One of the unusual characteristics of olanzapine is its low potency in producing Parkinsonian side effects and akathisia in humans. Olanzapine is a clozapine-like drug with respect to its chemical structure, its broad receptor affinity profile (dopamine $\mathrm{D}_{1}-\mathrm{D}_{5}$, serotonin $5 \mathrm{HT}_{2 \mathrm{~A}}, 5 \mathrm{HT}_{2 \mathrm{C}}$, $5 \mathrm{HT}_{6,1} \alpha$ noradrenergic, muscarinic cholinergic, and histamine $\left.\mathrm{H}_{1}\right)$; moreover, it shows selective action on the mesolimbic (A10) versus the nigrostriatal (A9) dopamine neurons, with chronic dosing (Skarsfeldt 1995), fails to stimulate Fos protein in dorsal striatum (Robertson and Fibiger 1996), and does not cause catalepsy.

Sertindole is a new antipsychotic drug jointly developed by Abbott Laboratories and Lundbeck A/S, a phenyl indole derivative (Sanchez et al. 1991). Like olanzapine, it has potent antipsychotic actions in schizophrenic psychosis, on both positive and negative symptoms (Zimbroff et al. 1997). Similarly, one of the unusual characteristics of sertindole is its low potency in producing Parkinsonian side effects and akathisia in humans. The drugs is clozapine-like in its selective action on A10 (mesolimbic), compared to A9 (nigrostriatal) dopamine neurons (Skarsfeldt 1992); indeed, this was the selection principle used prospectively in its design. Consistent with this anatomic selectivity, sertindole fails to stimulate Fos protein in dorsal striatum (Fink-Jensen and Kristensen 1994), and does not cause catalepsy at relevant doses (Sanchez et al. 1991). Despite this, its chemical structure and receptor profile differ from that of clozapine, with a considerably narrower receptor affinity profile (the dopamine $\mathrm{D}_{2}$ family, serotonin $5 \mathrm{HT}_{2 \mathrm{~A}}$, $5 \mathrm{HT}_{2 \mathrm{C}}$, and $\alpha_{1}$ noradrenergic receptors).

Based on the low incidence of Parkinsonism and akathisia with each of these antipsychotic drugs in clinical trials (Beasley et al. 1996; Zimbroff et al. 1997), and therefore, the potential that either drug might have a low incidence of TD with long-term use (Kane et al.
1986), we hypothesized that their potency in causing oral dyskinesias in rats with chronic dosing would also be low.

\section{METHODS}

Male Sprague-Dawley albino rats were divided into six treatment groups: Water ad libitum, $n=10$; haloperidol, $1.5 \mathrm{mg} / \mathrm{kg} /$ day, $n=10$; sertindole $0.5 \mathrm{mg} / \mathrm{kg} /$ day, $n=9$; sertindole, $2.0 \mathrm{mg} / \mathrm{kg} /$ day, $n=10$; olanzapine $0.5 \mathrm{mg} / \mathrm{kg} /$ day, $n=10$; and olanzapine $2.0 \mathrm{mg} / \mathrm{kg} /$ day, $n=10$. Because the doses of all the drugs are given in drinking water, they are administered gradually over the 24-hour period, not in a bolus. The dose of haloperidol was selected, because it has previously been used in our studies and reliably produces purposeless chewing movements (CMs). Moreover, we have previously demonstrated that this dose of haloperidol $(1.5 \mathrm{mg} / \mathrm{kg} /$ day $)$ produces rat drug plasma levels of $6.8 \pm 1.1 \mathrm{ng} / \mathrm{ml}$, which are in the clinical antipsychotic range (Gao et al. 1997). Plasma levels in this range in humans are associated with an incidence of TD of 5\% per treatment year (Kane et al. 1986).

For the new neuroleptics, we attempted to pick two doses of each that were in the range of this dose of haloperidol based on clinical potency and animal pharmacology. The recommended clinical dose ranges of haloperidol, olanzapine, and sertindole for schizophrenic psychosis are 8 to $16 \mathrm{mg}$ (VanPutten et al. 1991), 10 to 20 $\mathrm{mg}$ (Beasley et al. 1996), and 12 to $24 \mathrm{mg}$ (Zimbroff et al. 1997), respectively, with more uncertainty about the latter two dose ranges than that for haloperidol. Oral bioavailability in rat is substantial for all three drugs. The dose range selected for the new antipsychotics (0.5 and $2.0 \mathrm{mg} / \mathrm{kg}$ /day) spanned this range relative to haloperidol; moreover, plasma drug levels of all three drugs were analyzed in rat trunk blood.

Each drug was administered in drinking water continuously for 6 months. The two test drugs, sertindole and olanzapine, were separately dissolved in a minimum volume of glacial acetic acid. Each solution was diluted with distilled water and adjusted to $\mathrm{pH} 5.5$ to 6.0 , using $10 \mathrm{~N}$ sodium hydroxide, to give stock solutions of $33.3 \mathrm{mg} / 100 \mathrm{ml}$. The stocks were diluted every 7 days to $3.3 \mathrm{mg} / 100 \mathrm{ml}$ drug solution for the high-dose groups, and to $0.833 \mathrm{mg} / 100 \mathrm{ml}$ drug solution for the low-dose groups. Rats were housed two per cage; drug dosage was calculated (weekly initially; monthly in last months) by the amount of water drunk, assuming equal drinking between the two animals. Body weight was monitored. Haloperidol dosing in this strategy has been previously verified by us (Gao et al. 1997); preclinical scientists familiar with the pharmacology of olanzapine and sertindole verified the dosing strategy for the two new drugs (J. Arnt, personal communication; R. Lahti, 
personal communication). Drug concentration in the drinking water was adjusted as needed, through monitoring water intake, to maintain the target daily dose. After 6 months of continuous drug administration, rats were sacrificed by decapitation. Trunk blood was collected at sacrifice for measurement of plasma drug levels.

The animal preparation utilized here has been previously examined in detail in this laboratory. Under the conditions of this experiment in our laboratory, male Sprague-Dawley rats have a gradual onset of movements with haloperidol treatment. The rate of $\mathrm{CMs}$ after onset of haloperidol treatment, and the offset of movements, and the effects a single 5-mg acute dose of atropine on the CMs are given in Table 1 (Tamminga et al. 1990). Both Long-Evans animals and Wistar rats behaved differently from Sprague-Dawley rats with respect to these characteristics. After this initial descriptive experiment, we have applied this same metholodology in several subsequent experiments to test the pharmacologic characteristics of this animal paradigm (Shirakawa and Tamminga 1994; Kakigi et al. 1995; Gao et al. 1994; Gao and Tamminga 1996). We assume that the careful duplication of this animal preparation procedure within our laboratory will reproduce the behavioral characteristics of this paradigm.

Purposeless oral movements were assessed as previously described (Gunne et al. 1982; Tamminga et al. 1990). The CMs were counted for two 5 -minute periods by two trained raters blind to treatment group, with their ratings averaged; they were assessed at baseline, then monthly with treatment, and twice in the final treatment month. The two raters were the same throughout the study. The two end ratings were averaged for the final score at study end. Where ratings differed by more than $10 \%$, a third rating was done later, and all three were averaged. Rat CMs were analyzed in two ways: by a simple average of rat CM scores across treatment groups; and by a dichotomy into high-CM and low-CM groups. We have identified a score of $\geqslant 8$ $\mathrm{CMs} / 5 \mathrm{~min}$ by mixture analysis as the optimal criterion for dichotomization into high and low CMs (Hashimoto et al. 1998), and applied this criterion prospectively. We have previously argued that the high-CM group may be TD-like.

Trunk blood was collected at the time of sacrifice, and the plasma was frozen at $-20^{\circ} \mathrm{C}$ until analysis. Haloperidol was quantified using a modification of the method of Bianchetti and Morselli (1978), using chlorohaloperidol as the internal standard and simultaneously determining reduced haloperidol in the laboratory of Thomas Cooper (Nathan Klein Institute). Olanzapine levels were analyzed by Lilly Research Laboratories using high-performance liquid chromatography (HPLC) with electrochemical detection (Method LC-FID-PM-P-21, 1995). Sertindole levels were analyzed by Abbott Laboratories using an ethyl acetate: hexane (80:20) extract of the samples followed by
Table 1. Characteristics of Haloperidol (Hal)-Induced CMs (\# per $2 \mathrm{~min}$ ): Time to Onset, Offset; Atropine Effect (Tamminga et al. 1990)

\begin{tabular}{lcccc}
\hline CM onset time & 1 Week & 4 Weeks & 8 Weeks & 12 Weeks \\
Hal & 4.2 & 5.8 & 13.8 & 14.2 \\
Water & 1.0 & 1.4 & 2.0 & 1.8 \\
CM offset time & On & $1 \mathrm{dd}$ & $10 \mathrm{dd}$ & $15 \mathrm{dd}$ \\
Hal & 15.3 & 10.3 & 10.6 & 6.6 \\
Water & 0.5 & 2.0 & 3.0 & 2.0 \\
CM/atropine resp. & $0 \mathrm{~min}$ & $30 \mathrm{~min}$ & \multicolumn{2}{c}{$60 \mathrm{~min}$} \\
Atropine (5 mg/dose) & 15.4 & 9.8 & \multicolumn{2}{c}{15.6} \\
\hline
\end{tabular}

tandem mass spectrometric detection of the reconstituted extract (Assay S96-002, NB 26262, 1995).

A one-way analysis of variance (ANOVA) with Dunnett's post-hoc test was used to analyze the data. First, the placebo group was used as the control group, and then the haloperidol group was the designated control group for the Dunnett's analysis. Because of the potential influence of outliers, the analyses were repeated with nonparametric comparisons. The results were the same. In addition, a chi-square analysis was carried out with the frequency data with significance tested using the two-tailed Fisher's exact test. Effect sizes were calculated for each test drug group in comparison to haloperidol.

\section{RESULTS}

\section{Incidence of Purposeless Chewing Movements}

After 6 months of treatment, water alone did not result in any "high" CM animals in the control animals; whereas, haloperidol induced these oral movements in $60 \%$ of the animals. The two doses of olanzapine both induced a 10\% incidence of high CMs. The two doses of sertindole induced a $0 \%$ (lower dose) and 10\% (higher dose) incidence of high CMs in the treated animals, respectively (Figure 1). The chi-square analysis of all six groups comparing high versus low CMs was significant at $p=.00184$. Individual comparisons between the test drug dose groups and haloperidol using chi-square analysis showed a strong trend for a difference between each dose of olanzapine and haloperidol and the lower dose of sertindole and haloperidol (each comparison, $p=$ .057); whereas, the higher dose of sertindole was significantly different from haloperidol $(p=.011)$. The effect sizes for the difference between each test drug group and haloperidol are moderate-to-large and are specified in Table 2.

\section{Rates of Purposeless Chewing Movements and Plasma Drug Levels}

Average rates of CMs for all treatment groups are given in Table 2 and graphed individually in the figure. Using 


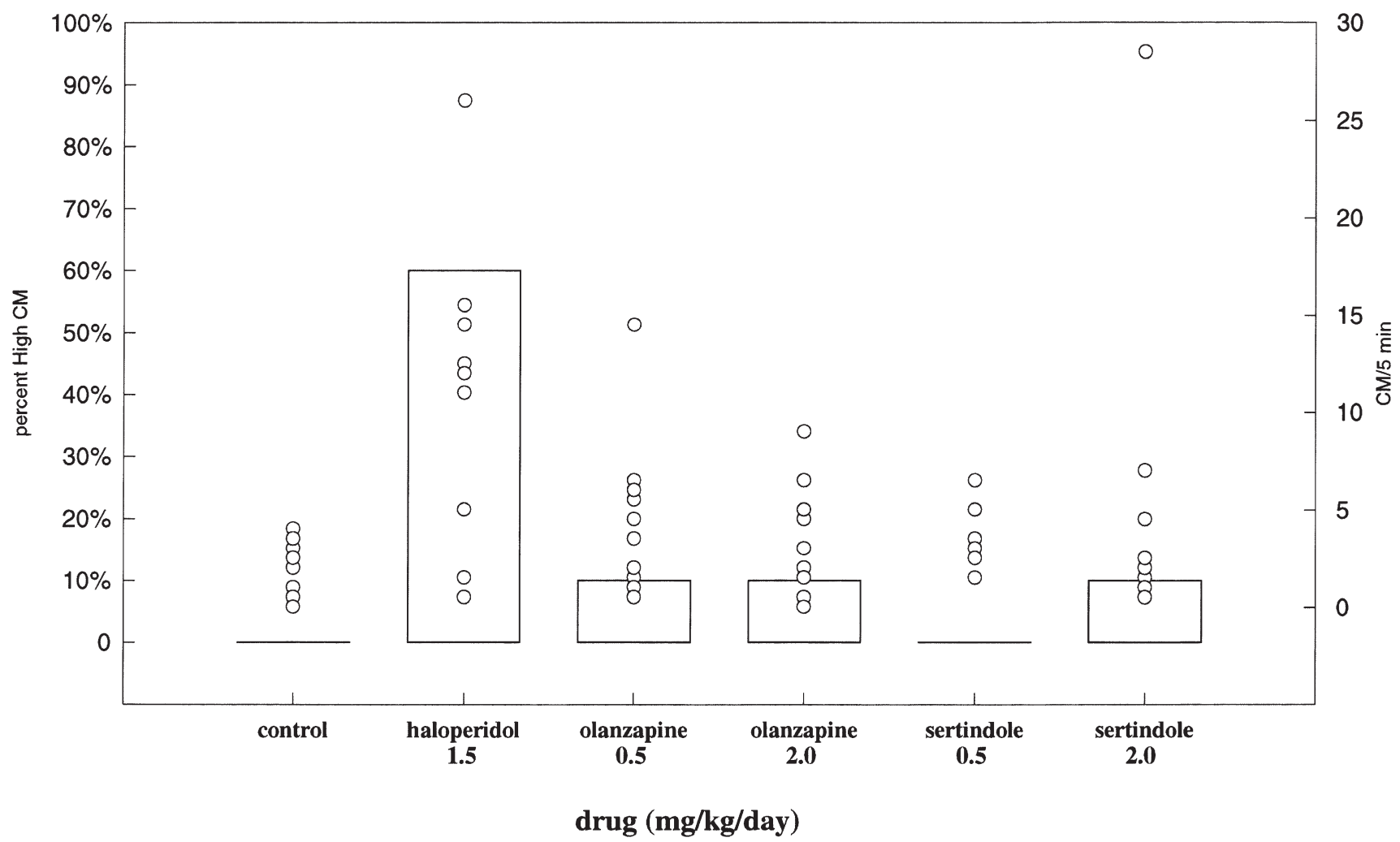

Figure 1. The percentage of animals in each treatment group with high levels of purposeless chewing movements $(>8 \mathrm{CM} / 5$ $\mathrm{min}$ ) is shown in the open columns and keyed on the left vertical axis. The open circles represent the individual levels of $\mathrm{CM}$ for each individual rat in each treatment group and are keyed on the right vertical axis. In the haloperidol-treated animals, both the percentage high $\mathrm{CM}$ and individual $\mathrm{CM}$ were higher than the control group. Neither measure was different from control in the any of the other treament groups.

ANOVA, CM rates in the haloperidol group differed from placebo $(p<.05)$, but CM rates of the study drugs did not differ from placebo at either dose level using Dunnett's post-hoc test. Sertindole at its lower dose produced significantly fewer CMs than haloperidol $(p<.05)$.

Plasma drug concentrations in each drug treatment group are indicated in Table 2 . The average haloperidol plasma concentration in the rat plasma is within the human therapeutic range for haloperidol $(4-16 \mathrm{ng} / \mathrm{ml})$.
The same is true for olanzapine (10-20 $\mathrm{ng} / \mathrm{ml})$ and for sertindole $(30-100 \mathrm{ng} / \mathrm{ml})$, although the therapeutic range of each of these drugs is less well established.

\section{DISCUSSION}

These data show that, in contrast to the traditional antipsychotic drug haloperidol, the two new antipsychotics,

Table 2. Plasma Drug Concentrations

\begin{tabular}{lcccc}
\hline Drug (mg/kg/day) & Number & $\begin{array}{c}\text { CM Rate }(\mathbf{X} \pm \text { SD) } \\
\text { (CMs/5 min) }\end{array}$ & $\begin{array}{c}\text { Effect size } \\
\text { (haloperidol } \\
\text { comparison) }\end{array}$ & $\begin{array}{c}\text { Drug Plasma } \\
\text { Level (ng/ml) }\end{array}$ \\
\hline Water & 8 & $2 \pm 1.5$ & - & - \\
Haloperidol (1.5) & 9 & $10 \pm 8$ & - & $13 \pm 10.1$ \\
Olanzapine (0.5) & 10 & $4.6 \pm 4.1$ & .794 & $1.5 \pm 1.7$ \\
Olanzapine (2.0) & 9 & $3.9 \pm 2.9$ & .910 & $9.3 \pm 7.4$ \\
Sertindole (0.5) & 6 & $3.3 \pm 1.6$ & 1.00 & $39.4 \pm 14$ \\
Sertindole (2.0) & 8 & $5.1 \pm 8.4$ & .583 & $218.9 \pm 42$ \\
\hline
\end{tabular}

This table specifies the average rate of rat oral dyskinesias (as $\mathrm{CM} / 5 \mathrm{~min}$ ) for each neuroleptic drug in the middle column and the effect size of its difference from haloperidol in the next column. Dose is listed in the left column. Plasma levels of each drug are listed in the right column. 
olanzapine and sertindole, do not produce oral dyskinesias in rats in the high-CM range, when administered over 6 months. Neither new drug produced CM rates significantly different from placebo, and their incidence of high CMs was significantly different from haloperidol or showed a strong trend for this effect $(p=.057)$; moreover, the effect sizes of the test drug group comparisons to haloperidol are substantial. The motor response produced by these two new drugs mimics results with chronic clozapine administration in rats (Gunne et al. 1982; Kakigi et al. 1995). It is not entirely surprising that these two drugs demonstrate the same action on oral dyskinesias as clozapine, based upon their similar regional depolarization blockade of dopamine neurons, Fos activation pattern, and clinical motor side effect profile, despite their very different receptor affinity profiles (Bymaster et al. 1996; Sanchez et al. 1991).

The preclinical characteristic shared by olanzapine and sertindole that may predict their low motor side effect profile in human studies is their anatomic selectivity demonstrated in the depolarization blockade model (Grace et al. 1997) and using Fos protein activation (Robertson et al. 1994). Both olanzapine and sertindole relatively selectively affect dopamine neurons that innervate the limbic and frontal cortices (the A10 dopamine neurons) and leave relatively unaffected those dopamine neurons involved in the striatum (the A9 dopamine neurons) (Skarsfeldt 1995). Although the full meaning of this model has been debated, the preclinical data indicate a preferential corticolimbic localization of drug action and a limited action in regions of striatum associated primarily with motor function. We, as well as others, have proposed that this selective action is the basis for clozapine's low incidence of acute motor side effects and tardive dyskinesia in humans (Gao et al. 1997). Moreover, as with clozapine, in the nonhuman primate, neither olanzapine nor sertindole causes acute dystonic reactions in the neuroleptic-primed monkeys at dose levels comparable to human treatment doses (Casey 1996). The primate data suggest that, at effective antipsychotic doses, clozapine, olanzapine, and sertindole do not act strongly enough on CNS motor pathways to produce acute motor side effects; therefore, these drugs might lack late motor side effects as well.

Clinical data from long-term studies with olanzapine suggest that treatment-emergent dyskinesias may occur at a significantly lower rate with olanzapine than with haloperidol. Several blinded extension protocols attached to Lilly's multicenter olanzapine versus haloperidol efficacy studies included 904 previously neuroleptic-treated schizophrenic individuals participating for 6 to 150 weeks (median drug exposure with haloperidol was 203 days; with olanzapine, 237 days). At study start, participants did not evidence any dyskinesias; at study end, $4.6 \%$ of the group of 197 haloperidol-treated patients demonstrated treatment-emergent dyskinesias by the strictest criteria, and $1 \%$ of the 707 olanzapinetreated subjects demonstrated the dyskinesias (Tollefson et al. 1997). Thus, the rate of "treatment-emergent" dyskinesias in rats with olanzapine as compared to haloperidol is consistent with this piece of clinical information about the relative incidence of neurolepticinduced dyskinesias with olanzapine in humans.

Based not on the receptor profile of these two drugs, but on their preclinical pharmacologic property of selective A10 dopamine neuronal action and distribution of Fos protein activation, we predicted a low incidence of oral dyskinesias in rats with chronic treatment. We have confirmed this prediction; moreover, the low dyskinesia rate in rats with both new drugs correlates with a low rate of acute motor side effects with acute human use and with early clinical information of low treatment-emergent dyskinesia incidence with olanzapine.

These pieces of data, considered together, can support considerable optimism about the low motor side effect profile of these two new antipsychotic drugs in human use. Definitive clinical studies have already demonstrated their low acute Parkinsonism profile. The rat oral dyskinesia study reported here predicts a low TD incidence with their chronic use, to the extent that this rat condition can be seen to model human TD. Future prospective TD incidence studies with olanzapine and sertindole will test this prediction.

\section{ACKNOWLEDGMENTS}

This work was supported by NIMH Grant \#MH49667.

\section{REFERENCES}

Beasley CM, Jr., Sanger T, Satterlee W, Tollefson G, Tran P, Hamilton S (1996): Olanzapine versus placebo: Results of a double-blind, fixed-dose olanzapine trial. Psychopharmacology 124:159-167

Bianchetti G, Morselli PI (1978): Rapid and sensitive method for determination of haloperidol in human samples using nitrogen-phosphorous selection detection. J Chromatog 153:203-210

Bymaster FP, Calligaro DO, Falcone JF, Marsh RD, Moore NA, Tye NC, Seeman P, Wong DT (1996): Radioreceptor binding profile of the atypical antipsychotic olanzapine. Neuropsychopharmacology 14:87-96

Casey DE (1996): Behavioral effects of sertindole, risperidone, clozapine, and haloperidol in Cebus monkeys. Psychopharmacology 124:134-140

Clow A, Theodorou A, Jenner P, Marsden CD (1980): Changes in cerebral dopamine function induced by a year's administration of trifluoperazine or thioridazine and their subsequent withdrawal. Adv Biochem Psychopharmacol 24:335-340

Ellison G, See R, Levin E, Kinney J (1987): Tremorous mouth 
movements in rats administered chronic neuroleptics. Psychopharmacology 92:122-126

Fink-Jensen A, Kristensen P (1994): Effects of typical and atypical neuroleptics on Fos protein expression in the rat forebrain. Neurosci Lett 182:115-118

Gao X-M, Kakigi T, Friedman MB, Tamminga CA (1994): Tiagabine inhibits haloperidol-induced oral dyskinesias in rats. J Neu Transm 95:63-69

Gao X-M, Tamminga CA (1996): Phencyclidine produces changes in NMDA and kainate receptor binding in rat hippocampus over a 48 hour time course. Synapse 23:274-279

Gao XM, Hashimoto T, Cooper TB, Tamminga CA (1997): The dose-response characteristics of rat oral dyskinesias with chronic haloperidol or clozapine administration. J Neural Transm 104:97-104

Glenthoj B, Hemmingsen R (1989): Intermittent neuroleptic treatment induces long-lasting abnormal mouthing in the rat. Eur J Pharmacol 164:393-396

Grace AA, Bunney BS, Moore H, Todd CL (1997): Dopamine-cell depolarization block as a model for the therapeutic actions of antipsychotic drugs. Trends Neurosci 20:31-37

Gunne LM, Growdon J, Glaeser B (1982): Oral dyskinesia in rats following brain lesions and neuroleptic drug administration. Psychopharmacology 77:134-139

Hashimoto T, Ross DE, Gao XM, Tamminga CA (1998): Mixture in the distribution of haloperidol-induced oral dyskinesias in the rat supports an animal model of tardive dyskinesia. Psychopharmacology 137:107-112

Kakigi T, Gao XM, Tamminga CA (1995): Drug-induced oral dyskinesias in rats after traditional and new neuroleptics. J Neural Transm 101:41-49

Kane JM, Woerner M, Borenstein M, Wegner J, Liberman J (1986): Integrating incidence and prevalence of tardive dyskinesia. Psychopharmacol Bull 254-258

McKinney WT, Bunney WE (1969): Animal models of depression. Arch Gen Psychiat 21:240-248

Robertson GS, Fibiger HC (1996): Effects of olanzapine on regional c-fos expression in rat forebrain. Neuropsychopharmacology 14:105-110

Robertson GS, Matsumura H, Fibiger HC (1994): Induction patterns of Fos-like immunoreactivity in the forebrain as predictors of atypical antipsychotic activity. J Pharmacol Exp Therapeu 271:1058-1066

Sanchez C, Arnt J, Dragsted N, Hyttel J, Lembol HL, Meier E (1991): Neurochemical and in vivo pharmacological profile of sertindole, a limbic-selective neuroleptic compound. Drug Devel Res 22:239-250

Shirakawa O, Tamminga CA (1994): Basal ganglia GABA and dopamine $\mathrm{D}_{1}$ binding site correlates of haloperidolinduced oral dyskinesias in rat: Implications for the mechanism of tardive dyskinesia. Exper Neurol 127:62-69

Skarsfeldt T (1992): Electrophysiological profile of the new atypical neuroleptic, sertindole, on midbrain dopamine neurons in rats: Acute and repeated treatment. Synapse 10:25-33

Skarsfeldt T (1995): Differential effects of repeated administration of novel antipsychotic drugs on the activity of midbrain dopamine neurons in the rat. Eur J Pharmacol 281:289-294

Tamminga CA, Dale JM, Goodman L, Kaneda H, Kaneda N (1990): Neuroleptic-induced vacuous chewing movements as an animal model of tardive dyskinesia: A study in three rat strains. Psychopharmacology 102:474-478

Tollefson GD, Beasley CM, Jr, Tamura RN, Tran PV, Potvin JH (1997): Blind, controlled, long-term study of the comparative incidence of treatment-emergent tardive dyskinesia with olanzapine or haloperidol. Am J Psychiat 154:1248-1254

VanPutten T, Marder SR, Wirshing WC, Aravagiri M, Chabert N (1991): Neuroleptic plasma levels. Schizophr Bull $17: 197-216$

Waddington JL (1990): Spontaneous orofacial movements induced in rodents by very long-term neuroleptic drug administration: Phenomenology, pathophysiology, and putative relationship to tardive dyskinesia. Psychopharmacology 101:431-447

Weiss JM, Kilts CD (1995): Animal models of depression and schizophrenia. In Schatzberg AF, Nemeroff CV (eds), Textbook of Psychopharmacology. Washington, DC, American Psychiatric Press, pp 81-123

Zimbroff DL, Kane JM, Tamminga CA, Daniel DG, Mack RJ, Wozniak PJ, Sebree TB, Wallin BA, Kashkin KB, The Sertindole Study Group (1997): A controlled, doseresponse study of sertindole and haloperidol in schizophrenia. Am J Psychiat 154:782-791 